\title{
Labyrinthe
}

$6 \mid 2000$

Numéro 6

\section{De l'espace frontalier au territoire transfrontalier}

\section{Olivier Denert et Harold Hurel}

\section{OpenEdition}

Journals

Édition électronique

URL : http://journals.openedition.org/labyrinthe/429

DOI : $10.4000 /$ labyrinthe.429

ISSN : 1950-6031

Éditeur

Hermann

Édition imprimée

Date de publication : 1 juin 2000

Pagination : 141-148

Référence électronique

Olivier Denert et Harold Hurel, « De l'espace frontalier au territoire transfrontalier », Labyrinthe [En ligne], 6 | 2000, mis en ligne le 23 mars 2005, consulté le 19 avril 2019. URL : http://journals.openedition.org/ labyrinthe/429; DOI : 10.4000/labyrinthe.429

Ce document a été généré automatiquement le 19 avril 2019

Propriété intellectuelle 


\title{
De l'espace frontalier au territoire transfrontalier
}

\author{
Olivier Denert et Harold Hurel
}

Depuis le début de cette année universitaire, Labyrinthe organise chaque mois un séminaire d'ouverture destiné aux étudiants de première année du Centre de Formation des Journalistes (CFJ). Nous vous proposons ici un rapide compte rendu de la seconde de ces interventions.

1 Situées à la croisée d'enjeux idéologiques, politiques et territoriaux, les frontières et les espaces qu'elles déterminent ont une signification qui dépasse largement leur emprise territoriale limitée. Ces espaces de marge incarnent en effet avec une acuité particulière les mutations du territoire dans un contexte de construction européenne, d'affirmation des régions et de remise en cause des modèles nationaux centralisateurs. En effet, c'est aux frontières que la construction européenne apparaît plus clairement qu'ailleurs comme une construction territoriale. La libre circulation des biens et des personnes y crée dans les faits de nouveaux territoires, marquant ainsi un changement d'échelle et un renversement complet de perspective.

2 Silencieuses, lentes et dispersées, ces mutations n'en constituent pas moins une révolution au sens le plus propre du terme. Appartenant au « temps long " défini par Fernand Braudel, elles closent un cycle séculaire de construction du territoire et constituent les prémisses d'une nouvelle époque. Aujourd'hui à un moment charnière, on voit une conception strictement hexagonale du territoire céder la place à une conception davantage communautaire, non sans conflits et concurrences.

La frontière est donc un espace marginal sur lequel s'est jouée de manière particulièrement explicite la construction nationale, dans ses dimensions territoriales et idéologique. Corrélativement, c'est également sur cet espace que se joue aujourd'hui la construction européenne et que des espaces frontaliers sont appelés à devenir des territoires transfrontaliers. Jacques Delors avait parfaitement compris cet enjeu en affirmant que « Les régions frontalières sont les laboratoires de l'Europe ». 
De l'hexagone à l'Europe : changement d'échelle et renversement de perspectiveLe prisme hexagonal

4 L'analogie entre le territoire français et la figure de l'hexagone est emblématique d'une conception idéale, géométrique et isolée du territoire. Modélisée en géographie dans les années 1960 par l'École de Roger Brunet, cette figure constitue certainement l'ultime avatar d'une conception du territoire née pendant le siècle de Louis XIV et réaffirmée dans la construction de l'État-Nation au XIX ${ }^{\mathrm{e}}$ siècle. Dans sa rationalité et sa géométrie l'hexagone n'est d'ailleurs pas sans rappeler le jardin à la française du xvII ${ }^{\mathrm{e}}$ siècle. Par sa prétention à la pureté minérale et à la modélisation scientifique, il marque la prégnance de cette représentation du territoire et de l'étendue des mutations que nous abordons. Sans vouloir faire un usage anachronique de cette figure, on peut néanmoins la considérer comme l'aboutissement d'une construction idéologique, politique, administrative et technique $\mathrm{du}$ territoire. Structure mentale puissante dans l'appréhension que nous avons de l'espace français, elle constitue un mode opératoire de l'administration et de l'aménagement du territoire.

5 Depuis plus de trois siècles, le territoire français a été construit de manière militaire et diplomatique mais aussi administrative et technique, marquant ainsi profondément l'espace et les mentalités. Quelques repères jalonnent cette construction : les grands traités du siècle de Louis XIV, qui définissent nombre de frontières actuelles, la carte de Cassini (1744), qui donne pour la première fois une vision unifiée du pays ou encore la départementalisation, œuvre de la Constituante, qui ordonne l'administration du territoire. La constitution d'un réseau routier (création du corps des Ponts et Chaussées en 1746) puis d'un réseau ferroviaire au XIX ${ }^{e}$ siècle (réseau en étoile de l'ingénieur Legrand) consacre l'unification technique du territoire.

La dimension communautaire

6 Longtemps implicite et exclusif, le mode hexagonal de production du territoire est aujourd'hui remis en cause, tant dans la pratique que dans les schémas de pensée. Un espace européen intégré émerge avec la libre circulation des biens et des personnes ou la disparition des contrôles douaniers dans l'espace Shengen. La politique communautaire intègre cette nouvelle dimension en mettant en place le Schéma Directeur de L'Espace Communautaire (SDEC), tentative encore modeste et indicative de coordonner le développement de l'espace communautaire. La fragilité de ce processus et la prudence des institutions européennes s'expriment bien dans le terme même " d'espace ", préféré à celui de « territoire » aux résonances trop volontaristes.

7 La constitution d'un espace européen n'est toutefois pas contradictoire avec l'existence des territoires nationaux. Elle vient plus souvent compléter ces échelles que les remplacer, conforme en cela au principe de subsidiarité qui voudrait que les fonctions territoriales soient affectées aux échelles les plus à même de les assumer.

De l'espace frontalier au territoire transfrontalier Les frontières dans l'ordre national

Limites du territoire, les frontières le définissent. Elles constituent ainsi le lieu privilégié de sa construction dans l'ordre national et de ses mutations actuelles. La définition du mot même de frontière est emblématique. Selon Lucien Febvre et Daniel Nordman, le terme $\mathrm{a} a u \mathrm{XVI}^{\mathrm{e}}$ et au XVII ${ }^{\mathrm{e}}$ siècle une définition avant tout militaire. La frontière est alors le front défini par une armée et constitue ainsi un cas particulier de la limite, terme général employé dans la diplomatie pour fixer les partages entre deux souverainetés. La frontière a un enjeu militaire et elle définit les limites par la force de manière unilatérale. 
La limite incarne au contraire un mode bilatéral et négocié de définition des limites territoriales. Les deux termes deviennent synonymes au $\mathrm{xIX}^{\mathrm{e}}$ siècle, marquant la militarisation des limites territoriales avec l'avènement des États-Nations.

La frontière est ainsi une construction idéologique au fort contenu symbolique. Au XVII siècle la signature fastueuse du Traité des Pyrénées par Louis XIV sur l'île des Faisans, au milieu du fleuve frontière de la Bidassoa, est la célébration d'un nouvel ordre territorial et étatique. Cet ordre prétend trouver ses fondements dans la nature même en s'appuyant par de minutieux bornages sur les fleuves et les lignes de crêtes. Le mythe des frontières naturelles qui tente de justifier une construction idéologique et des limites arbitraires dans la nature s'en trouve ainsi fondée. Il prospérera au XIX ${ }^{e}$ siècle avec l'avènement des États-Nations.

10 À la construction idéologique de la frontière est ainsi étroitement associée une construction concrète dans l'espace. La frontière n'est plus une zone aux allégeances incertaines mais un fait géographique, objectivement observable. Cette construction est avant tout militaire, la frontière étant un espace dédié à la défense du territoire. Cette spécialisation fonctionnelle s'imprime durablement dans le territoire, des forteresses de Vauban à la ligne Maginot, mais aussi dans les mentalités, en particulier avec l'avènement de la conscription. Cette spécialisation est exclusive et contribue à l'enclavement des frontières, espaces périphériques souvent éloignés des centres de décisions.

11 Cependant les régions frontalières se situent aux limites territoriales et idéologiques de l'État-Nation. Elles le définissent mais en incarnent aussi les limites, montrant le caractère artificiel, culturel et parfois arbitraire de cette construction.

Vers des territoires transfrontaliers

12 Le renversement de perspective territoriale avec l'émergence d'une échelle communautaire met encore une fois les espaces frontaliers en première ligne. Plus qu'ailleurs, il signifie un renversement dans les fonctions et la définition de ces espaces. L'espace frontalier orienté à $180^{\circ}$ devient un territoire transfrontalier orienté à $360^{\circ}$. La frontière cesse d'être strictement une ligne pour devenir un espace transitoire d'amplitude variable entre deux ordres nationaux.

13 L'émergence de territoires transfrontaliers se traduit de manière politique et territoriale. Ces espaces naguère périphériques et subordonnés se recentrent et trouvent les moyens de leur gestion, notamment en utilisant les possibilités offertes aux régions par les lois de décentralisation. De nouvelles unités apparaissent, et de grandes agglomérations étendent leur rayonnement au-delà des frontières. Les massifs montagneux ne s'arrêtent plus aux lignes de crêtes, mais sont envisagés dans leur globalité. Cessant d'être des champs de bataille potentiels et ouverts à de nouveaux horizons, ces territoires trouvent les moyens d'un nouveau développement économique. Prenant conscience de l'unité des espaces situés de part et d'autre de la frontière, les habitants et leurs élus les dotent de moyens d'agir et d'exister. De nombreux projets de coopération voient le jour, portés par des structures juridique inédites.

Deux perspectives concurrentes sur un même espace

14 L'émergence de territoires transfrontaliers ne se fait pas sans difficultés et sans conflits. Deux perspectives s'affrontent et coexistent aujourd'hui : l'une héritée du passé, mais forte d'une inertie accumulée considérable, l'autre se projetant dans l'avenir, mais encore ténue et parfois contradictoire. La construction territoriale héritée des États-Nations est 
forte, structurée, dotée d'une puissante inertie. Elle est encore très largement dominante, même si elle n'est désormais plus seule.

Cette situation engendre de nombreuses contradictions dans l'action des différents services de l'État dans les régions frontalières. La question des traités régissant la coopération transfrontalière est à cet égard emblématique. Ceux-ci, quand ils existent, ont en effet des portées fort différentes selon les États avec lesquels ils ont été conclus. Cela a pour conséquence une inégalité de traitement des différentes frontières françaises, l'Alsace pouvant faire ce qui est refusé au Nord-Pas-de-Calais, et met à mal la fiction d'un État jacobin traitant de manière égale toutes ses composantes territoriales.

Instruments et réalités du développement transfrontalier

Depuis le début des années 1990, on assiste à la création de nombreux instruments de différente nature pour répondre à la montée en puissance de la problématique transfrontalière.

Le programme INTERREG

17 Le programme INTERREG finance des projets transfrontaliers au titre de la politique structurelle communautaire. Sa mise en œuvre donne lieu à des procédures complexes qui nuisent à son efficacité. Ainsi pour un seul projet, il existe deux financements européens (reçus dans chacun des deux pays contractants) et deux contreparties nationales. Déléguée à l'État, la gestion d'INTERREG est souvent technocratique et unilatérale ce qui vient relativiser très largement la portée transfrontalière des actions.

Des améliorations notables sont cependant apportées aujourd'hui à ce programme, montrant ainsi un certain assouplissement de l'État. La gestion du programme est davantage confiée aux collectivités locales (Départements ou Régions) et elle est envisagée de manière plus transfrontalière, avec une réelle concertation des partenaires nationaux. Même si INTERREG a été pour partie dévoyé au profit de l'Équipement unilatéral des territoires frontaliers, il a eu au moins le mérite de forcer certaines Régions et États à définir des politiques transfrontalières.

Des évolutions législatives

En dépit de ses interrogations et de ses contradictions, l'État a parfois réellement innové pour faire avancer la problématique transfrontalière. Ainsi de nouveaux outils juridiques ont été créés à l'occasion de traités internationaux (traité de Bayonne avec l'Espagne en 1995, traité de Karlsruhe avec l'Allemagne, le Grand Duché du Luxembourg et la Suisse en 1996). C'est ainsi qu'a vu le jour le Groupement Local de Coopération Transfrontalière, structure qui en assurant la gestion ou la réalisation commune d'équipements publics pourrait permettre la naissance de nouvelles formes de territoire, venant compléter les échelles actuelles. Cela dit, on assiste à une certaine contradiction de l'État sur ce projet. Tout en ayant signé des traités, il freine voire bloque l'instruction des demandes locales pour la création de ces nouvelles structures.

On assiste également à l'émergence d'une nouvelle échelle territoriale, le Pays, qui pourrait profiter aux régions frontalières rurales et leur permettre de se structurer. $\mathrm{Ce}$ territoire traditionnel rappelant les découpages de l'Ancien Régime est une échelle de projet censée mieux cerner les bassins de vie et être aux plus près des réalités quotidiennes. Même s'ils ne constituent pas un nouvel échelon administratif, l'État craint parfois y voir une menace pour les départements.

Différentes avancées législatives vont encore permettre des avancées dans la coopération transfrontalière. Ainsi la loi sur les Sociétés d'Économie Mixte rend désormais possible la 
participation à part égale des collectivités françaises et étrangères dans le capital de sociétés d'économie mixte. Cette disposition facilite la maitrise d'ouvrage commune et binationale en matière d'aménagement et d'urbanisme.

À l'inverse, d'autres lois dans un but de protection du territoire nationale prennent une acception absurde dans le cas du transfrontalier. Ainsi, la loi sur les déchets interdisant de faire traiter les déchets empêche toute gestion de cette question à l'échelle de l'agglomération transfrontalière.

Les avancées et les reculs en matière de coopération transfrontalière sont très liés aux hésitations de l'État, inquiet de rompre encore un peu plus avec le centralisme traditionnel et redoutant de laisser la porte ouverte à un démantèlement de l'État-Nation. Ces hésitations sont d'autant plus grandes qu'elles se situent sur le terrain conflictuel du degré d'autonomie des collectivités locales et de leur capacité à gérer leurs relations avec des partenaires étrangers. Ce conflit est d'autant plus exacerbé que la structure des États voisins de la France est parfois fédérale et accorde une grande marge de manœuvre à leurs collectivités sur la question transfrontalière.

L'effet frontière

24 Le fait transfrontalier s'illustre par différents phénomènes géographiques, économiques et sociaux que ce soit par l'existence d'agglomérations, de flux de travailleurs frontaliers ou de pratiques commerciales. La frontière joue ainsi souvent un rôle de catalyseur et de générateur de flux en raison du différentiel de réglementation et de coûts, créant ainsi un véritable effet-frontière. Il est même probable que si la frontière disparaissait, on assisterait à une normalisation et une certaine dévitalisation de zones frontières, qui perdraient cette spécificité dynamique. Ainsi 210000 personnes résidant en France franchissent quotidiennement les frontières pour aller travailler dans les pays voisins alors qu'elles sont moins de 10000 à faire le trajet inverse. La différence des coûts fonciers incite de nombreux citoyens Suisses et Allemands à venir résider en Alsace. Les entreprises tentent de gagner sur plusieurs tableaux en jouant sur les prix de l'immobilier, la fiscalité, le coût de la main d'œuvre et le droit du travail.

Le fait transfrontalier concentre ses plus fortes dynamiques au sein des corridors que sont les espaces urbains frontaliers. Cette nouvelle échelle d'agglomération concerne quelques des plus grandes villes françaises comme Lille, Strasbourg, Nice ou Dunkerque mais aussi des villes étrangères comme Bâle et Genève.

L'émergence des territoires transfrontaliers constitue un chantier de longue haleine qui viendra profondément modifier la notion de frontière sans toutefois la faire disparaître. Il serait en effet illusoire de croire que la libre circulation peut effacer les ordres administratifs, politiques, culturels ou techniques qui trouvent leurs limites sur ces frontières. Cependant, les frontières nous interrogent aujourd'hui comme par le passé sur la nature du territoire. Les valeurs et les formes qu'elles prendront au sein de l'espace communautaire le définiront donc largement. 


\section{BIBLIOGRAPHIE}

NORDMAN Daniel, Frontières de France, Paris, NRF, Gallimard 1998 ; « Des limites d'État aux frontières nationales ", Les lieux de Mémoire, sous la direction de Pierre NORA, Paris, NRF, Gallimard 1986.

UEBERSCHLAG Jean, La Coopération transfrontalière, Rapport au Premier Ministre, 1996.

\section{AUTEURS}

\section{OLIVIER DENERT}

Olivier Denert est chargé de mission à la Mission Opérationnelle Transfrontalière. denertmot(at)post.club-internet.fr

HAROLD HUREL

Harold Hurel est chargé de mission en urbanisme et aménagement. harold.hurel (at)caramail.com 